\title{
Direct Dynamic Protein-Affinity Selection Mass-Spectrometry
}

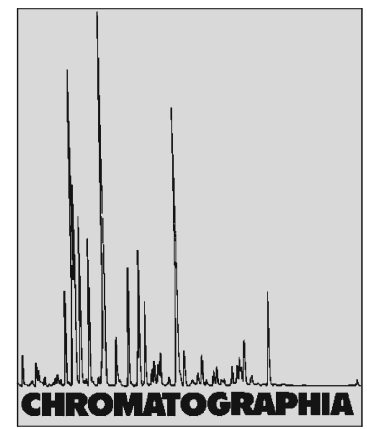

2010, 72, 7-13

\author{
Niels Jonker, Henk Lingeman ${ }^{\bowtie}$, Hubertus Irth \\ BioMolecular Analysis Group, Department of Chemistry, Faculty of Sciences, VU University Amsterdam, De Boelelaan 1083, \\ 1081 HV Amsterdam, The Netherlands; E-Mail: h.lingeman@few.vu.nl
}

\begin{abstract}
A new methodology is described enabling the affinity screening of potential ligands towards the human estrogen receptor alpha ligand binding domain (ER $\alpha-L B D)$. In-solution incubation is performed of the analyte and the His-tagged ER $\alpha$-LBD. The bound complex is immobilized on a nickel-loaded protein-affinity selection column, where after the unbound fraction is removed. The immobilized protein-ligand complex is exposed to a decreased $\mathrm{pH}$ value and an increased organic modifier concentration releasing the ligand for MS detection, and precipitating the proteins on a filter positioned between the affinity column and the mass spectrometer. The trapping column can be regenerated for reuse at least 70 times. The advantages of the methodology over existing methodologies are the absence of a preconcentration as well as a chromatographic separation step, resulting in a significantly shorter analysis time compared to previously described procedures, and in addition, allowing the determination of solutes with unfavorable chromatographic properties. The overall analysis time now can be reduced about $250 \%$ to approximately $6 \mathrm{~min}$. Replacing the filters after every measurement results in an intra-day standard deviation of $14.8 \%$ and an inter-day standard deviation of $21.3 \%$.
\end{abstract}

\section{Keywords}

Column liquid chromatography

Protein affinity

Ligand screening

Dynamic immobilization

His-tagging

Estrogen receptor

\section{Introduction}

Dynamic protein affinity selection (SPELC-MS) has been developed for finding new pharmacologically active compounds. This tool can be used as a screening approach for large numbers of compounds for their affinity towards a certain target protein $[1,2]$. The main advantages over the already existing methods and procedures are the reduced analysis time, the absence of a reporter ligand and the ability to detect weak affinity binders, thus reducing the number of false negatives to a minimum [3-6]. However, in order to enable analyzing these compounds pre-concentration on a reversed-phase solid-phase extraction (SPE) cartridge is necessary followed by a suitable liquid chromatographic (LC) separation. The disadvantage is that not always suitable LC systems are available for all individual compounds especially when compounds with widely varying physico-chemical properties are present in the mixture.

Recently, significant progress has been made in the multi-component synthesis of functional small heterocycles, resulting in a variety of new compound classes that might possess biological 


\begin{tabular}{|c|c|c|c|c|c|}
\hline \multirow[t]{3}{*}{ Structure } & Substance & {$[\mathrm{M}+\mathrm{H}]$} & $\log P$ & pKa & Structure \\
\hline & BG168 & 382.16 & 2.24 & - & \\
\hline & NEL382A & 232.15 & 0.19 & 9.28 & \\
\hline & NEL216BC & 231.12 & 1.02 & 2.11 & \\
\hline & NEL269A & 353.19 & 3.12 & 7.13 & \\
\hline & NEL235C & 274.15 & 2.78 & 12.73 & \\
\hline & NEL285 & 368.16 & 3.21 & 7.50 & \\
\hline & BG164 & 446.19 & 5.90 & 10.63 & \\
\hline & DHP & 351.14 & 5.26 & 4.14 & \\
\hline & NEL296A & 273.16 & 2.86 & 9.25 & \\
\hline & NEL216 & 378.22 & 3.10 & 8.28 & \\
\hline & NEL362B & 320.24 & 1.60 & 5.72 & \\
\hline & NEL350 & 288.21 & 2.79 & 6.82 & \\
\hline & MM-III-12-2E & 497.13 & 5.66 & 11.35 & \\
\hline & MM-III-16-2C & 404.12 & 4.05 & 4.16 & \\
\hline & VUA036 & 309.14 & 4.88 & 5.76 & \\
\hline & VUA028 & 321.13 & 2.91 & 11.25 & \\
\hline & MP84 & 355.18 & 7.04 & 3.20 & \\
\hline & MP186 & 292.1 & 1.51 & 10.62 & \\
\hline & MP161 & 481.23 & 6.68 & 2.94 & \\
\hline
\end{tabular}

Fig. 1. All compounds in the testset. All data calculated using ACDLabs software

activity [7-9]. A few classes of these small heterocycles have previously shown affinity for the estrogen receptor [10-12]. In order to evaluate the affinity of these compounds towards ER $\alpha$-LBD, a test set of these solutes was analyzed using the previously reported dynamic protein-affinity chromatography (DPAC)-SPE-LC-MS (mass spectrometry) methodology [1]. As mentioned, some of the analytes are possessing poor chromatographic properties resulting in low recoveries, and as a result unfavorable sensitivities, of the SPE-LC-MS approach compared to a direct-injection LC-MS technique tool. In order to widen the applicability, a new method, direct protein affinity-selection mass spectrometry, has been developed. In this case the bound ligand is directly eluted from the affinity column to the
MS, without the need for SPE pre-concentration and/or a chromatographic separation.

This methodology has been developed using a commercially available online SPE-LC (Symbiosys Pharma) system and both cartridge clamps are used inline. The first one contains the nickelcoated protein-affinity selection column and the second one contains a simple filter cartridge. The model protein, $\mathrm{ER} \alpha-\mathrm{LBD}$, is His-tagged, which allows immobilization on the nickel cartridge due to the nickel-His-tag interaction. A real challenge in the system optimization consists of finding the proper balance between the use of additives to improve protein stability and the resulting signalto-noise ratio in MS. Furthermore, optimization of the transfer of the analyte mixture from the autosampler to the affinity column, and subsequent separation of the bound and unbound fraction proved to be a critical step in the whole procedure. The resulting system was able to determine all compounds in the test set as well as a group of five known estrogens which were serving as positive controls.

\section{Experimental}

\section{Materials}

ELISA blocking reagent (EBR) was purchased from Roche Diagnostics (Pensburg, Germany). 17 $\beta$-Estradiol, diclofenac, testosterone, nicotin, epibatidine, equol, potassium chloride, monosodium dihydrogenphosphate and disodium monohydrogenphosphate were obtained from Sigma (Schnelldorf, Germany). Diethylstilbestrol, warfarin and sodium chloride came from Riedel de Haen (Seelze, Germany). Ethylenediaminetetraacetic acid was received from Acros (Geel, Belgium), while comestrol was from Fluka (Buchs, Switzerland). Nickel nitrate came from Aldrich (Steinheim, Germany) and acetonitrile, methanol (both LC-MS grade) as well as formic acid were from Biosolve (Valkenswaard, The Netherlands). All reagents were at the highest quality available. Water was produced by a Milli-Q device of Millipore (Amsterdam, The Netherlands).

The test set contained the following compounds, synthesized in house and provided by the Section of Synthetic and Bio-organic Chemistry: BG168 [13], NEL3282A [14], NEL216BC [15], NEL269A [15], NEL235C [15], NEL285 [15], BG164 [16], DHP [17], NEL296A [15], NEL216 [15], NEL362B[14], NEL350 [14], MM-III-16-2C [18], VUA036 [19], VUA028 [19], MP84 [17], MP186 [20], MP161 [21]. The structures, molecular weights and some relevant physico-chemical properties of the compounds are given in Fig. 1.

\section{System Setup}

The system setup is shown in Fig. 2. It consists of a Symbiosys Pharma (Spark Holland, Emmen, The Netherlands) 
sample pre-treatment system which is equipped with an LC20AD gradient LC system (Shimadzu, Kyoto, Japan). Valves 1 and 4 as shown in Fig. 1 are integral parts of the automated cartridge exchange (ACE) component of the symbiosis. In the presented methodology, they are not switched, but merely used to route the flow from the LC pumps towards the clamps containing the nickel cartridge (valve 1) and the filter cartridge (valve 4). In Fig. 2, a is the loading configuration, in which the protein-ligand complex is immobilized on the cartridge, and all other components are flushed towards the waste. Simultaneously, pump 2 conditions the filter cartridge for use in configuration B. After simultaneously switching valves 2 and 3 , the system is in elution configuration (b in Fig. 2). Pump 2 is switched offline, and the nickel-cartridge is online with the LC gradient system, the filter cartridge and the mass spectrometer, allowing detection of the bound ligand. The mass spectrometer used is a Thermo Electron (Breda, The Netherlands) LCQ Deca ion trap MS, operated in the ESI + and APCI-mode.

\section{Protein-Ligand Incubation}

The His-tagged human estrogen receptor ligand binding domain was cultured inhouse according to the procedure described by Eiler et al. [22]. This, except for the fact that a modified binding buffer was used, consisted of $10 \mathrm{mM}$ phosphate buffered saline (PBS) with a $\mathrm{pH}$ of 7.4 and $1 \mathrm{mg} \mathrm{mL}^{-1}$ EBR. The final concentration of $\mathrm{ER} \alpha$ was determined to be $933 \pm 50 \mathrm{nM}$. The results were corrected for any activity loss caused by protein degradation at $4{ }^{\circ} \mathrm{C}$ (autosampler temperature).

A 15 min incubation with $1 \mu \mathrm{M}$ solution of one of the analytes in the ER $\alpha$ stock solution was performed at ambient temperature, in a $1.8 \mathrm{~mL}(32 \times 11.6 \mathrm{~mm})$ autosampler vial, while slowly shaken in an electronically modified vortex (VWR International, Amsterdam, The Netherlands). The incubation mixture was transferred to the Reliance (Spark Holland, Emmen, The Netherlands) autosampler and stored at $4{ }^{\circ} \mathrm{C}$.
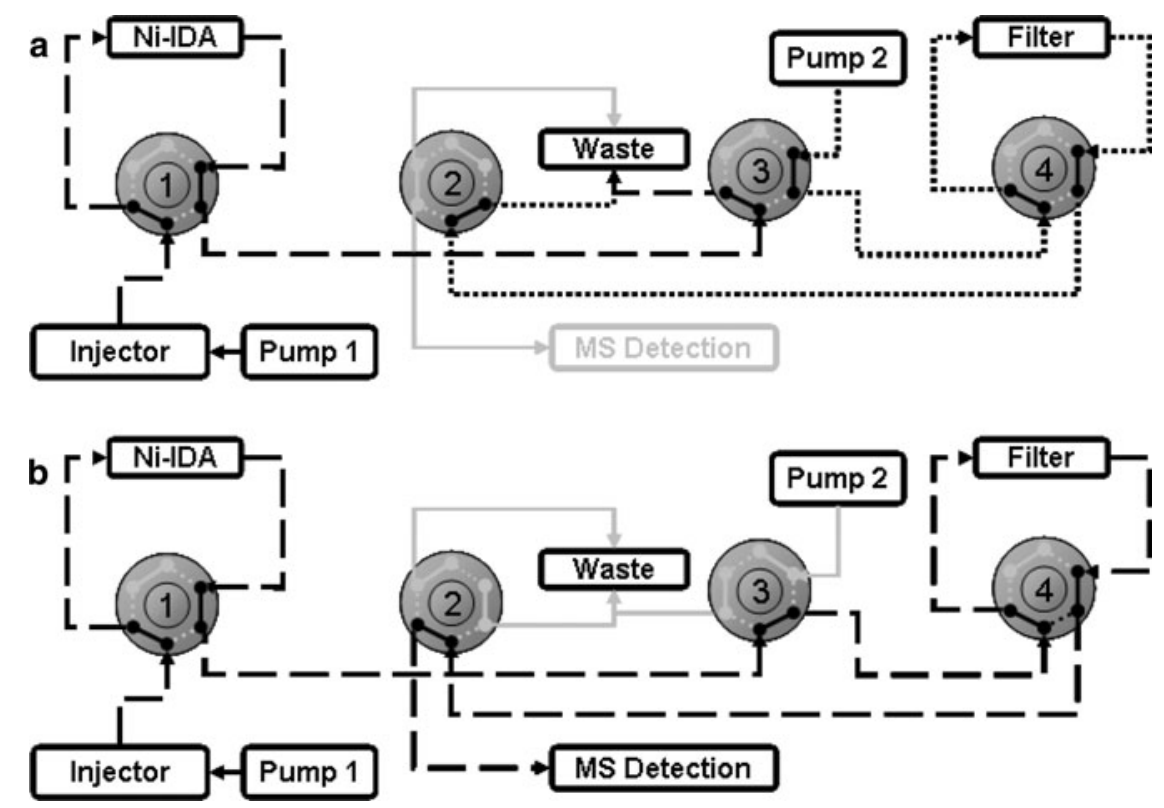

Fig. 2. a Cartridge conditioning and immobilization. Pump 1 conditions the left cartridge clamp in the symbiosis with binding buffer, and transports the injection plug to the clamp for immobilization, followed by a wash step. Pump 2 conditions the filter cartridge in clamp 2 with elution buffer. b Ligand elution and MS detection. Both symbiosis cartridge clamps are used online. Pump 1 pumps elution buffer through both cartridges, eluting the bound ligand to the mass spectrometer for detection, and precipitating the protein on the filter. After the measurement both cartridges are replaced. The nickel cartridge is stored for regeneration, the filter cartridge is disposed

\section{Protein-Affinity Selection}

A $100 \mu \mathrm{L}$ volume of the incubation mixture was injected into a plug of $150 \mu \mathrm{L}$ of binding buffer and transported to the protein-affinity selection cartridge. This cartridge $(10 \times 2 \mathrm{~mm}$ ID) was packed with $50-80 \mu \mathrm{m}$ silica particles coated with iminodiacetate and loaded with $\mathrm{Ni}^{2+}$-ions. The material was purchased from Silicycle (Quebec City, Canada) and has been custom packed by Spark Holland. The $\mathrm{Ni}^{2+}$-ions form a strong non-covalent bond with any His-tagged protein, allowing efficiently immobilizing the protein on the cartridge. The particles were relatively large (50-80 $\mu \mathrm{m})$ which preventing the cartridge from getting clogged. Immobilization conditions were adjusted to retain protein-ligand complex stability upon immobilization on the cartridge via the nickel-His-tag interactions. Non-specific binding to the sorbent material was reduced to a minimum by conditioning the cartridge with the binding buffer before injection. A short wash step using $1 \mathrm{~mL}$ of washing buffer [PBS at $\mathrm{pH} 7.4$ containing $5 \%(v / v)$ of acetonitrile] removed the non-bound ligand from the cartridge at $1 \mathrm{~mL} \mathrm{~min}{ }^{-1}$. Subsequently, the filter is switched online with the cartridge, where after it is desorbed using $800 \mu \mathrm{L}$ of the elution buffer [90\% $(v / v)$ acetonitrile in water containing $0.2 \%(v /$ $v$ ) of formic acid]. As a result, the bound ligand was eluted to the MS, while the protein precipitated on the filter. Several types of filters were tested, amongst them commercially available $\mathrm{C} 2, \mathrm{C} 8$ and $\mathrm{C} 18$ SPE cartridges developed specifically for use in the symbiosis (Spark Holland), as well as a number of in-house packed cartridges containing molecular sieve material (60-500 $\mu \mathrm{m}$ diameter, 10-30 ̊ pore size) combined with stainless steel shields with three different pore sizes (1, 2 and $3 \mu \mathrm{m}$ ). A new filter was used for every measurement. In the case of some of the positive controls (i.e. estradiol, equol, diethylstilbestrol) the APCI-mode was used and consequently no formic acid was added to the elution buffer. After elution, the nickel cartridge was regenerated by stripping the $\mathrm{Ni}^{2+}$-ions off the silica backbone with a $0.1 \mathrm{M}$ 


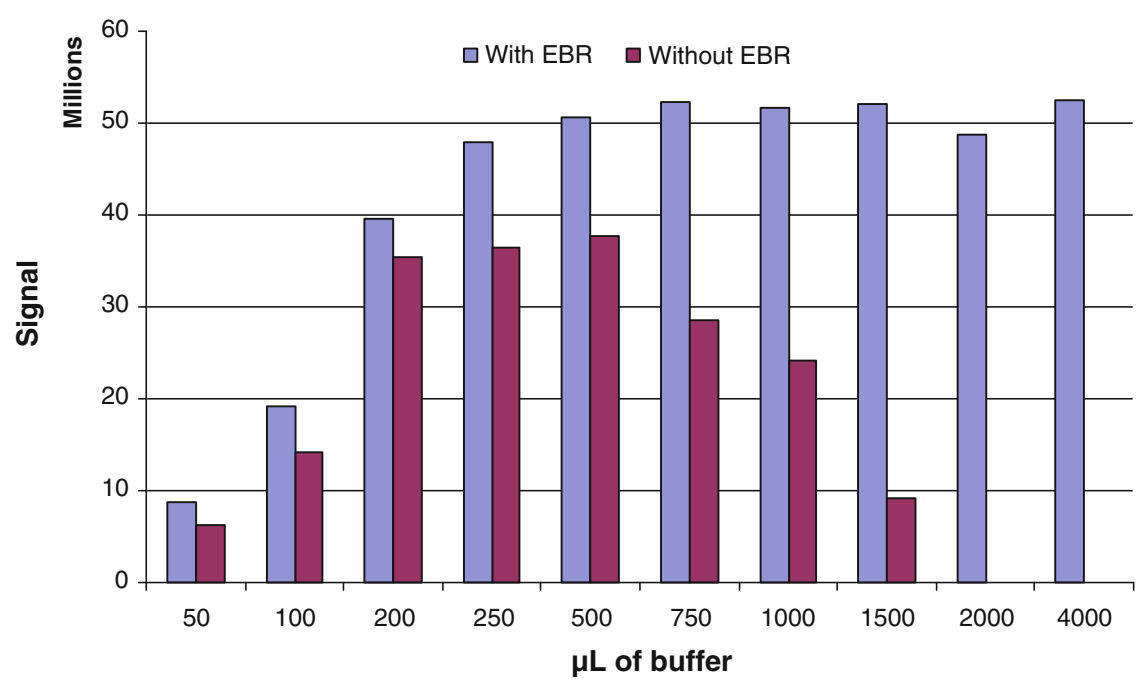

Fig. 3. Protein stability with and without EBR. For both experiments, the first $250 \mu \mathrm{L}$ were used to transport the injection plug to the affinity column. If the valve is switched before the first $250 \mu \mathrm{L}$ have been processed, not all of the injection volume has bound to the column, and some of the complex is not measured, thus decreasing the signal. After the transport step, a wash with EBR containing wash buffer keeps the complex intact for at least up to $4 \mathrm{~mL}$ of wash volume. However, in the wash buffer without EBR the complex remains stable for only around $250 \mu \mathrm{L}$ of wash volume. At higher volumes, the complex falls apart, and the measured ligand decreases

solution of EDTA in water at $\mathrm{pH}$ 8.0. After two washing steps with subsequently ten column volumes of phosphate buffer and water, the column was reloaded by flushing it with ten column volumes of $1 \mathrm{mM} \mathrm{NiNO}$ solution.

\section{MS Detection}

The elution was performed at $500 \mu \mathrm{L}$ $\min ^{-1}$ in order to make the method as fast as possible, and the time settings in the LCQ Deca were optimized accordingly. The mass spectrometer was used both in the ESI + and the APCI- mode. In the ESI + mode, the capillary temperature and voltage were set to $250{ }^{\circ} \mathrm{C}$ and $+20 \mathrm{~V}$, respectively. The tube lens offset was $50 \mathrm{~V}$. The nitrogen flow rate was $15 \mathrm{~L} \mathrm{~min}^{-1}$ for the nebulizing gas and $5 \mathrm{~L} \mathrm{~min}^{-1}$ for the auxiliary gas. In the APCI- mode, a capillary temperature of $250{ }^{\circ} \mathrm{C}$ and a vaporizing temperature of $450{ }^{\circ} \mathrm{C}$ were used. The capillary voltage was set to $-50 \mathrm{~V}$ and the tube lens to $25 \mathrm{~V}$. The corona discharge current was set to $15 \mu \mathrm{A}$. The gas flows were identical to the $\mathrm{ESI}+$ mode.

\section{Results and Discussion}

The main factors to be optimized in the proposed system were the concentration of the protein stabilizing agents (e.g. EBR) in the washing buffer and the stability of the protein using MS compatible conditions. In addition, the speed and time of the wash step should be optimized in order to compensate for any occurring decrease in protein stability. The second part of the optimization process focuses on the elution step. The percentage of organic modifier in the elution buffer has to be optimized in combination with the use of a high flow rate and the proper MS settings for such flow rates. Other parameters to be optimized were the smallest transport volume enabling an efficient immobilization of the injection plug on the proteinaffinity selection cartridge, as well as the type of the filter cartridge used. Finally, the repeatability of the method has to be determined employing a filter cartridge and by using a new filter cartridge for every measurement. All optimization experiments have been performed using estradiol, the native ligand of ER $\alpha$ in the APCI - ionization mode.
Optimization of the Wash Step

The separation of the bound and unbound fraction of the ligand required a wash step. In order to keep the proteinligand complex as stable as possible the wash step would preferably be performed with the binding buffer, since this buffer is optimized to enable formation of the protein-ligand complex. For the stabilization of the protein-ligand complex the binding buffer contained EBR, which consisted of the proteolytic degradation products of purified gelatin. Using binding buffer in the wash step would result in one column volume (approximately $19 \mu \mathrm{L}$ ) of binding buffer being eluted to the filter and MS. Unfortunately, the presence of $1 \mathrm{mg}$ $\mathrm{mL}^{-1}$ of EBR in the binding buffer resulted in a significant decrease of the MS sensitivity. The decrease of the signal at a concentration of $1 \mathrm{mg} \mathrm{mL}^{-1}$ was about $100 \%$ (no analyte could be detected due to ion suppression effects) compared to a situation without EBR. Several washing buffers were tested containing lower concentrations of EBR, revealing that detection of the ligand was only possible at concentrations lower than $10 \mu \mathrm{g}$ $\mathrm{mL}^{-1}$. At this concentration the signal reduction was ca. $75 \%$ However, this concentration of EBR had almost no effect on the protein stability. The conclusion is that any concentration of EBR that is sufficiently low not to cause ion suppression in the mass spectrometer, no longer has a positive effect on protein stability. Thus, in order to allow direct elution to the mass spectrometer, the washing buffer should not contain any EBR, and the decreased protein-ligand complex stability should be factored in the experimental design.

As a result of this conclusion, a transport and binding step using EBRcontaining binding buffer, and a wash step in phosphate buffered saline have to be performed. Consequently, it is important to assess the stability of the protein-ligand complex with and without the presence of EBR. In order to do this, the volume of the wash step was varied, while keeping the flow rate constant at $250 \mu \mathrm{L} \min ^{-1}$ (Fig. 3). The volume shown in Fig. 3 represents only 
the wash step, after completion of transport and binding. Two conclusions can be drawn from Fig. 3. First of all, when less than $250 \mu \mathrm{L}$ of wash buffer was used, ion suppression is caused by the incomplete removal of the EBR present in the binding buffer, which leads to significant signal reduction.

Secondly, after the transport, when EBR-containing wash buffer was used, the protein-ligand complex remained stable for at least $4 \mathrm{~mL}$ of wash volume. However, without EBR, the stability started decreasing after $500 \mu \mathrm{L}$ of the total buffer volume. This means that a wash volume of $250 \mu \mathrm{L}$ can safely be used. This wash volume completely removes the EBR from the column, thus preventing ion suppression, and is not long enough to cause protein degradation and its resulting signal reduction.

\section{Optimization of the Elution Step}

Both acetonitrile and methanol were considered to be suitable organic modifiers for the elution buffer. Initial experiments clearly showed that both solvents provided a significant different background signal using MS detection. It seemed that by using acetonitrile the number of ion-suppressing compounds was higher. This assumption was supported by a precipitation experiment in which one or both solvents were added to a small amount of stock solution of $\mathrm{ER} \alpha$-LBD. The acetonitrile addition produced a clotted precipitate, while methanol addition resulted in a rather fine precipitate. As a result it is suspected that when using acetonitrile a larger amount of the proteins, present in the system, is precipitated on the filter, and cannot interfere with the MS detection.

In order to optimize the elution buffer, the concentration of acetonitrile was varied in $10 \%$ increments from 0 to $100 \%$ in water, for two model compounds, coumestrol in the ESI + ionization mode and estradiol in the APCImode. The signal of the analyte was increasing using higher concentrations of acetonitrile up to $90 \%$, but at $100 \%$ acetonitrile the signal was significantly lower. A possible explanation for this

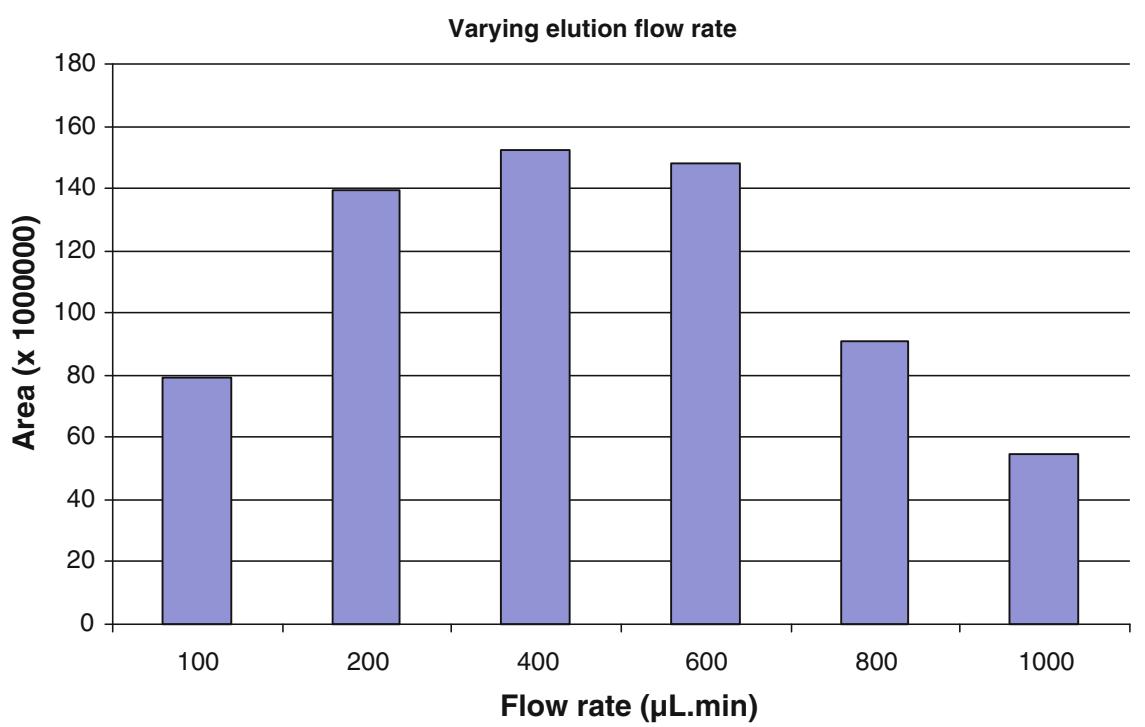

Fig. 4. Varying the elution flowrate. Ionization efficiency was optimal inbetween 400 and $600 \mu \mathrm{L}$ $\min ^{-1}$. As a result, a flow rate was chosen of $500 \mu \mathrm{L} \mathrm{min}{ }^{-1}$

phenomenon is the decreased ionization efficiency observed in the absence of water in the eluent. In order to speed up the process, the elution flow rate was varied between 200 and $1,000 \mu \mathrm{L} \mathrm{min}{ }^{-1}$. The data showed (Fig. 4) that the analyte signal was increasing at higher flow rates resulting in shorter analysis times. The limiting factor was the ionization efficiency of the MS. Using flow rates above $500 \mu \mathrm{L} \mathrm{min}{ }^{-1}$ caused the ionization efficiency to decrease, resulting in a reduced signal. As a result of these experiments a concentration of $90 \%$ $(v / v)$ of acetonitrile and a flow rate of

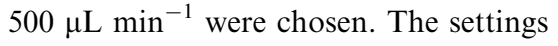
of the MS were optimized accordingly, resulting in the settings described above. Finally, because there is no retention of the solutes, the elution volume was set at twice the dead volume, resulting in a total volume of elution buffer of $800 \mu \mathrm{L}$.

\section{Cartridge Selection and Regeneration}

The applicability of several types of cartridges was tested for their usefulness as filter cartridges. In addition to the commercially available $\mathrm{C} 2, \mathrm{C} 8$ and $\mathrm{C} 18$ SPE cartridges of Spark Holland, inhouse developed cartridges packed with molecular sieves and equipped with stainless-steel shields with various pore sizes were tested. None of the cartridges showed any observable analyte binding at $90 \%(v / v)$ of acetonitrile. After visual inspection, the background signal obtained using the $\mathrm{C} 18$ cartridge as a filter showed the least interference of non-filtered compounds with the analytes eluting at the dead time. A second advantage of the $\mathrm{C} 18$ cartridges is that they are commercially available and more reliable compared with in-house packed cartridges.

When testing the repeatability of the system using a single $\mathrm{C} 18$ cartridge for a series of ten measurements, the standard deviation of the experiment was $33.8 \%$, and the signal was decreasing over time. The explanation of this effect was clogging of the filter cartridge, most likely by protein precipitation. Repeating the experiment using a new cartridge for every measurement resulted in an intra-day standard deviation of $14.8 \%$ $(n=10)$. The inter-day standard deviation for a period of 29 consecutive days was $21.3 \%$, which is only slightly higher than the previously published methodology that included a SPE wash and pre-concentration. Back flushing of the cartridges with a high concentration of an organic modifier did not regenerate the cartridges sufficiently, so the decision was made to replace the filter cartridges after every measurement. 


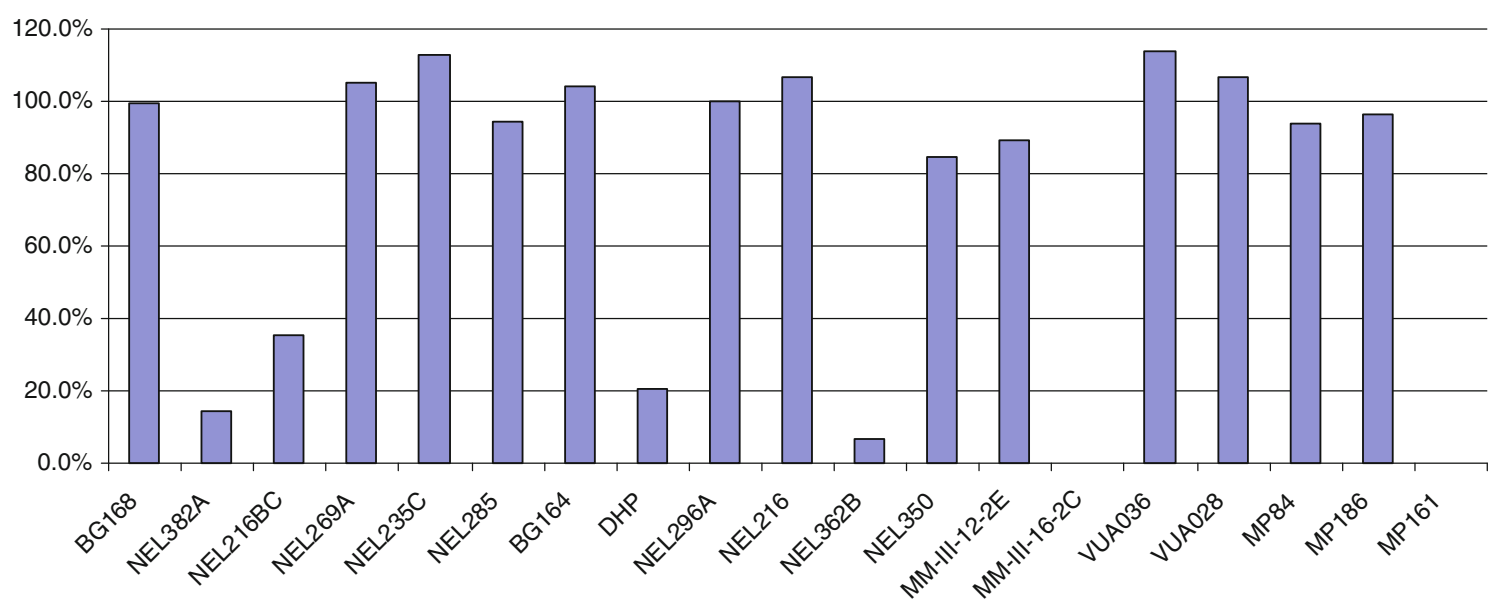

Fig. 5. SPE recovery. Results of the SPE-LC-MS recovery measurements. By definition $100 \%$ recovery means identical peak areas for a compound in SPE-LC-MS and direct injection measurements. Five compounds in this test-set show very poor recovery, caused by incomplete trapping of the compounds on the SPE cartridge

\section{Characterization of the Test Set}

The test set of heterocyclic compounds composed by our colleagues of the synthetic and bio-organic chemistry department consisted of compounds that were all detectable in the ESI + ionization mode. It was assumed the affinity of these compounds towards ER $\alpha$ could be assessed using the previously published DPAC-SPE-LC-MS system [1]. However, when routinely testing their chromatographic properties by comparing a direct injection LC-MS experiment with an SPE-LC-MS experiment, it was apparent some of the compounds in the test set showed very poor SPE recovery. Using the previously optimized MS settings, all compounds in the test set were detected using the direct injection-LCMS method. However, when the SPE trapping and wash were incorporated, for a small number of compounds, the signal decreased significantly. The results of these measurements are shown in Fig. 5. In most cases the recovery is around $100 \%$. However, for some of the compounds the recovery is rather low, which means that they cannot be determined with the SPE-based system. When cross-referencing the non-retained compounds with the ones listed in Fig. 1, the following explanations can be given. The poor recovery of the compounds NEL382A, NEL216BC and NEL362B can be explained because of their low log
$P$ values, while the $\mathrm{p} K_{\mathrm{a}}$ values of NEL216BC, MM-III-16-2C and MP161 indicate that a protonated nitrogen is present in the molecule at the acidic $\mathrm{pH}$ values used for protein-ligand complex dissociation. In the case of DHP no obvious explanation can be given. The overall conclusion is that using the given test set of compounds the previously described SPE-based methodology cannot be used to screen these compounds for affinity towards ER $\alpha$-LBD. In order to analyze these compounds, the methodology presented in this manuscript, in which bound ligands are eluted directly from the protein to the mass spectrometer, is the only viable option.

\section{Direct Protein Affinity Selection Measurements}

The optimized direct protein-affinity selection methodology as described above was used to measure the entire test set, as well as five positive controls (estradiol, coumestrol, equol, diethylstilbestrol and norethisterone) and five negative controls (warfarin, epibatidine, nicotin, testosterone and diclofenac). The results are shown in Fig. 6. The method successfully identified all five positive controls as binders to $\mathrm{ER} \alpha$ and all five negative controls as non-binders. In fact, the data obtained in the present system were fully comparable with the results obtained in the previously described SPE-based system [1]. Besides the controls, all of the compounds present in the test set were identified as nonbinders. When taking into account the statistically very small chance of finding a lead compound in a pharmaceutical library, this is not surprising. Furthermore, upon close inspection of the molecular structure of the compounds in the test set of in-house synthesized small heterocycles another possible reason for their non-binding was found. The previously mentioned small heterocycles that exhibit estrogenic activity, mostly have a hydroxyl group in similar locations to known estrogens. None of the compounds in our test set showed these moieties on the right locations, which suggested only a small chance of finding estrogenic activity for any of them. However, despite the fact that no binders could be identified in the test set, the methodology has been shown to successfully analyze the estrogenic activity for a wider range of analytes than previously published methodologies.

\section{Conclusions}

A direct protein-affinity selection mass spectrometry approach has been described as a viable alternative for the existing SPE- and/or LC-based procedures to determine protein-ligand affinity. It efficiently widens the range of analytes that can be screened using the protein-affinity selection methodology, by 


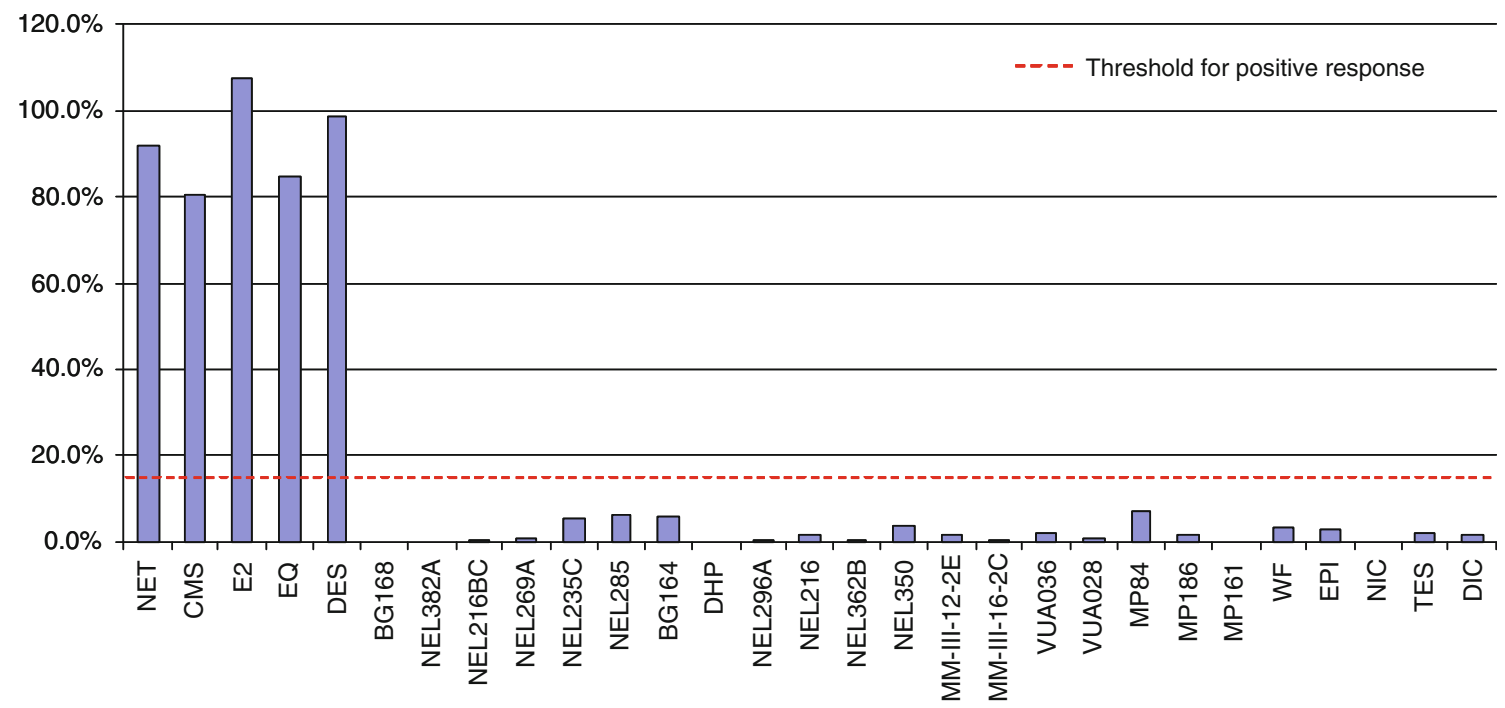

Fig. 6. Percentage of analyte bound to $\mathrm{ER} \alpha$. Peak area of the bound ligand compared with the direct injection. First five compounds are positive controls. All other compounds comprise the test set

removing the necessity for suitable chromatographic properties. Contrary to existing techniques the range of analytes that can be analyzed using direct dynamic protein affinity MS includes acidic, basic and amphoteric compounds as well as compounds showing irreversible adsorption to SPE material. A second advantage of the presented methodology is that the analysis time required for MS detection is reduced from $5 \mathrm{~min}$ to about $50 \mathrm{~s}$, because there is no need for LC separation and all compounds elute in the dead time. This reduction in analysis time, combined with the use of specialized equipment for fast sampling and miniaturized flow dimensions means that the system is compatible with existing high-throughput devices.

A limitation of the proposed method is the influence of protein stability enhancing additives to the binding buffer. Some of these additives can have a dramatic effect on the sensitivity of the MS detection, and thus cannot be used in the proposed setup. In order to assess whether a protein is suitable, the stability of the protein-ligand binding under MS compatible conditions must be considered for every individual protein.

\section{Acknowledgments}

The authors thank Eelco Ruijter, Maurice Mooijman, Niels Elders, Bas
Groenendaal, Rachel Scheffelaar and Danielle Vugts, all currently or recently working at the section of synthetic and bio-organic chemistry of the VU University in Amsterdam for their helpful discussions and assistance.

\section{Open Access}

This article is distributed under the terms of the Creative Commons Attribution Noncommercial License which permits any noncommercial use, distribution, and reproduction in any medium, provided the original author(s) and source are credited.

\section{References}

1. Jonker N, Kool J, Krabbe JG, Retra K, Lingeman H, Irth H (2008) J Chromatogr A $1205: 71$

2. Jonker N, Kretschmer A, Kool J, Fernandez A, Kloos D, Krabbe JG, Lingeman H, Irth H (2009) Anal Chem $81: 4263$

3. Choi Y, van Breemen RB (2008) Comb Chem High Throughput Screen 11:1

4. Ferrance JP (2007) J Chromatogr A 1165:86

5. Moaddel R, Lu LL, Baynham M, Wainer IW (2002) J Chromatogr B 768:41

6. Van Breemen RB, Huang CR, Nikolic D, Woodbury CP, Zhao YZ, Venton DL (1997) Anal Chem 69:2159

7. Elders N, van der Born D, Hendrickx LJD, Timmer BJJ, Krause A, Janssen E, de Kanter FJJ, Ruijter E, Orru RVA (2009) Angew Chem Int Ed 48:5856

8. Groenendaal B, Ruijter E, Orru RVA (2008) Chem Commun 5474

9. Scheffelaar R, Nijenhuis RAK, Paravidino M, Lutz M, Spek AL, Ehlers AW, de Kanter FJJ, Groen MB, Orru RVA, Ruijter E (2009) J Org Chem 74:660

10. Gust R, Keilitz R, Schmidt K, von Rauch M (2002) J Med Chem 45:3356

11. Laios I, Cleeren A, Leclercq G, Nonclercq D, Laurent G, Schlenk M, Wellner A, Gust R (2007) Biochem Pharmacol 74:1029

12. von Rauch M, Schlenk M, Gust R (2004) J Med Chem 47:915

13. Groenendaal B, Ruijter E, de Kanter FJJ, Lutz M, Spek AL, Orru RVA (2008) Org Biomol Chem 6:3158

14. Elders N, Ruijter E, de Kanter FJJ, Janssen E, Lutz M, Spek AL, Orru RVA (2009) Chem Euro J 15:6096

15. Elders N, Ruijter E, de Kanter FJJ, Groen MB, Orru RVA (2008) Chem Euro J 14:4961

16. Groenendaal B, Vugts DJ, Schmitz RF, de Kanter FJJ, Ruijter E, Groen MB, Orru RVA (2008) J Org Chem 73:719

17. Paravidino M, Bon RS, Scheffelaar R, Vugts DJ, Znabet A, Schmitz RF, de Kanter FJJ, Lutz M, Spek AL, Groen MB, Orru RVA (2006) Org Lett 8:5369

18. Manuscript in preparetion

19. Glasnov TN, Vugts DJ, Koningstein MM, Desai B, Fabian WMF, Orru RVA, Kappe CO (2006) QSAR Comb Sci 25:509

20. Unpublished data

21. Scheffelaar R, Paravidino M, Muilwijk D, Lutz M, Spek AL, de Kanter FJJ, Orru RVA, Ruijter E (2009) Org Lett 11:125

22. Eiler S, Gangloff M, Duclaud S, Moras D, Ruff M (2001) Protein Expr Purif 22:165 